\title{
Guillermo Schulz (1805-1877) y la Société Géologique de France
}

\author{
Pelayo González-Pumariega Solís ${ }^{(1)}$ y Luis Aurelio González Prieto ${ }^{(2)}$
}

(1) Escuela Politécnica de Mieres, Universidad de Oviedo, Gonzalo Gutiérrez s/n, 33600 Mieres (Asturias). pelayogs@uniovi.es

(2) Instituto de Educación Secundaria Rey Pelayo, Avenida Constantino González s/n, 33550 Cangas de Onís (Asturias). laureliogp@gmail.com

\begin{abstract}
RESUMEN
Las exigencias energéticas y productivas derivadas de la Revolución Industrial propiciaron que a finales del siglo XVIII la geología se empezara a consolidar como una disciplina científica independiente. La instauración de academias de minería y cátedras universitarias en varios países europeos permitió el desarrollo de las nuevas concepciones y teorías. Pero las asociaciones científicas también desempeñaron un papel fundamental en la creación de una comunidad geológica internacional, fomentando la difusión del conocimiento, los intercambios y encuentros. Así, tras la fundación de la pionera Geological Society of London en 1807, Francia creó la suya propia en 1830 y comenzó a publicar un boletín, que se convirtió rápidamente en un instrumento esencial para el desarrollo de la nueva ciencia. Guillermo Schulz (1805-1877) se formó como ingeniero de minas en la alemana Georg-August-Universität Göttingen. Tras ejercer la profesión durante cuatro años en las explotaciones de plomo de Almería, en 1830 se incorporó a la administración española como comisario de minas. Aparte de sus tareas facultativas, en 1832 la superioridad le encomendó elaborar una memoria y un mapa geognóstico de Galicia, y dos años después de Asturias. Para poder mantenerse al día de los rápidos avances experimentados por la geología, en 1833 se incorporó a la Société Géologique de France. A ella remitiría en primicia los resultados de sus investigaciones, así como varios de sus artículos. Con ello pretendía contribuir al progreso del conocimiento, así como atraer la atención de posibles inversores extranjeros. Schulz también colaboró con la asociación presentando a nuevos miembros.
\end{abstract}

Palabras clave: Geología, mapa geológico, mineral, Schulz, Société Géologique de France

\section{William Schulz (1805-1877) and the French Société Géologique}

\begin{abstract}
In the late eighteenth century, the energy and production demands produced by the Industrial Revolution determined that geology became established as an independent scientific discipline. The foundation of mining academies and university professorships in several European countries allowed the development of new concepts and theories. But scientific associations also played a key role in the creation of an international geological community, promoting the dissemination of knowledge, exchanges and meetings. Thus, after the establishment of the pioneering Geological Society of London in 1807, France created its own society in 1830 and began publishing a bulletin, which quickly became an essential tool for the development of the new science. William Schulz (1805-1877) trained as a mining engineer at the Georg-August-Universität in Göttingen. After working for four years in the lead mines of Almeria, in 1830 he became commissioner of mines for the Spanish administration. In 1832, apart from his facultative tasks, the General Directorate of Mines commanded him to prepare a report and a geognostic map of Galicia, and two years later of Asturias. In order to keep abreast of the rapid advances made in geology, he joined the French Geological Society in 1833. He was the first to send to the results of his research to the Society, as well as various articles. His aim was to contribute to the advancement of knowledge and attract the attention of potential foreign investors. Schulz also collaborated with the Society by presenting some of his friends and colleagues as new members.
\end{abstract}

Keywords: geological map, Geological Society of France, geology, mineral, Schulz 
González-Pumariega Solís, P. y González Prieto, L.A., 2017. Guillermo Schulz... Boletín Geológico y Minero, 128 (1): $221-240$

\section{ABRIDGED ENGLISH VERSION}

Geology was formed as an independent science in the late eighteenth century, once the scientists began to establish referential terms -formations and systems- properly constituted, classified and defined. In this process, the new energy and production demands for iron and coal produced by the Industrial Revolution were decisive, as they potentiated more active mining that required a theoretical apparatus able to predict where to find ore.

The British scientific community played an important role in the development of this new discipline, creating the Geological Society of London in 1807. Revolutionary France did not want to fall behind in geological studies, thus, chairs of mineralogy were created in prestigious public institutions, such as the Musée National d'Histoire Naturelle and the Institut National des Sciences et des Arts. In 1811 Georges Cuvier (17691832) and Alexandre Brongniart (1770-1847) published an essay on the mineralogical geography around Paris, which enumerated the various geological formations according to their overlap, lithology and fossils. The text was accompanied by two cross sections and a geognostic chart at a scale of 1:200.000, which is considered the first modern French geologic map.

Meanwhile, Spain was suffering a deep crisis and a great scientific backwardness, so that the first attempt to form a geologic map of the country did not take place until 1830, after the creation, five years previously, of the General Directorate of Mines by Fausto de Elhuyar (1755-1833).

\section{The birth of the Geological Society of France}

In 1828, a group of French naturalists found it necessary to create a geological association similar to the British, who were able to publish a journal dedicated to the divulgation of news and discoveries that were taking place in this emerging discipline. So, on March 17, 1830, Louis-Constant Prévost (1787-1856) with the collaboration, amongst others, of Ami Boué (1794-1881), Louis Cordier (1777-1861), Henri Marie Ducrotay of Blainville (1777-1850), Baron Férussac (1786-1836), etc., established the Société Géologique de France. Its overall objective was to contribute to the advancement of geology and, more specifically, to publicizing the national territory.

From the moment of its creation, the association developed sessions and special meetings to discuss the major advances and geological discoveries. In addition, it had a particularly effective means of dissemination, the Bulletin of the Geological Society of France, which on a monthly basis and an ordinary edition of five hundred copies were sent free of charge to all its partners (Fig. 1).

\section{Contributions of William Schulz to the Geological Society of France}

From Spain, the German-born mining engineer William Schulz (1805-1877), who began keeping a close relationship with the French association. Schulz had attended college at the Georg-August-Universität in Göttingen and in 1830, after working for four years in the lead mines of Almeria, he became commissioner of mines for the Spanish administration (Fig. 2). Apart from his facultative tasks, two years later the general manager ordered him to prepare a report and a geognostic map of Galicia. To address this work, he needed to keep abreast of the rapid advances made in geology, so in March 18, 1833, he joined the Geological Society of France, being backed by the then acting secretary Jules Desnoyers (1800-1887) and Boué, member of the Management Board (Fig. 3).

On May 30, 1844, Schulz managed to send an advance of his Descripción geognóstica del Reino de Galicia to the association, which gave a comprehensive report on the meeting held on July 7 (Fig. 4). In addition, Boué mentioned the conclusion of Schulz's fieldwork in his Résumé des progrés des Sciences Géologiques in1833 and the contents of his report was extracted by Claude-Antoine Rozet (1798-1858) in the Resume des travaux de la Société Géologique de France, et des progrès de la géologie en France, depuis le $1^{\text {er }}$ novembre 1833, jusqu'au $1^{\text {er }}$ novembre 1834.

Once this task was completed, he was immediately commissioned to compose a geognostic description and a petrographic map of Asturias (R.O. December 4, 1834). Although this work was delayed for two decades due to different causes, Schulz was promptly sending information on his progress to the Society. Thus, his first communication, entitled D'une notice abrégée géognostique sur les Asturies, was presented by board member Émile Le Puillon of Boblaye (1792-1843) on September 3, 1837 (Fig. 5).

Shortly afterwards, in the autumn of 1838 the Marquess of the Guadalquivir Marshes (1784-1842) commissioned Nicolas-Armand Buvignier (1808-1880) and François-Clément Sauvage (1814-1872) to carry out an facultative recognition of their newly acquired coalfields in Langreo and Siero. During their stay in Asturias, 
both engineers were able to contact Schulz and as a result of this relationship, Buvignier submitted a report to the association entitled Note géologique sur les Asturias on February 4, 1839. This article is especially notable for the five schematic geological cross sections made to show the relative position of the different terrains observed, as they are the first to include all the formations recognized at that time in the province, although with the characteristic simplicity of the time as regards tectonics (Fig. 6). Buvignier recognized that these sketches were to a great extent, "a result of my conversations with Mr. Schulz, rather than my own observations".

On the other hand, during his time as general inspector of the Real Cuerpo Facultativo de Minas, Schulz continued to send to the library of the association most of the reprints, books and works that he published in Spain. In addition, some members of the society, such as Edouard de Verneuil (1805-1873), Adolphe d'Archiac (1802-1868), Antoine-Edouard Collomb (1801-1875) and Antoine-Adrien Paillette (1809-1858) also made laudatory mentions of the activities developed by the German in his writings which had appeared in the Bulletin (Fig. 7).

In 1849 Schulz wrote in collaboration with Paillette a paper entitled Notice sur une pyrite stannifère (Ballesterosite) et sur quelques gisements d'etain en Espagne, that the French presented personally to the Geological Society in the meeting held on November 19 (Fig. 8). With this report, the two engineers notified the scientific community about the discovery of a new mineral -stannifer piryte-in the province of Lugo. Its publication led to a scientific and historic replica by the engineer Agustín Martínez Alcibar (1810-1872). Probably, this led to Schulz not signing an article on ancient mining that he had prepared with his friend and which two years later was presented by Paillette to the Geological Society of France under the title Recherches sur I'histoire et les conditions de gisement des mines d'or dans le nord de l'Espagne (Fig. 9).

Besides his scientific contributions, Schulz also played a role as host of new members in the Society. Thus, the first candidate presented by Schulz, along with the then secretary d'Archiac, was the professor of Chemistry applied to the Arts at the University of Oviedo, León Pérez de Salmeán (1810-1893), an event that took place at the meeting on April 2, 1838. Later, on June 21, 1841, he supported the Wallon mining engineer Jean Louis Armand Nagelmackers (1808-1891), who from 1833 had worked for 18 years at the major coal mining companies of Asturias. Other members that were sponsored by Schulz were the engineers of the Real Cuerpo Facultativo de Minas Policarpo Cía (1817-1867) and Joaquin Luaces (?-1847), who were admitted at the meeting of February 16, 1846. Finally, in January 6, 1851, he introduced two of his best friends: the colonel and metallurgical engineer Francisco Antonio Elorza (1798-1873), who led Fábrica de Armas de Trubia (Asturias) for 20 years and in 1852 participated with Schulz in creating a textile mill in Vivero (Lugo); and the Belgian mining engineer Adolphe Jean Charles Desoignie (1816-1898), who spent his entire professional life in Asturias and was named alternate testamentary executor by the German.

\section{Conclusions}

Schulz's relationship with the Geological Society of France has hardly been treated by his biographers, despite being very important for the development of his scientific and professional activity. With it, he adapted their knowledge and observations in the advances in geology, successfully addressing the development of geological descriptions and petrographic maps of the provinces of Galicia and Asturias. In return, he sent to the association the results of his investigations, revealing the geological formation of the peninsular northwest and contributed significantly to the progress of this science. In addition, Schulz promoted the development of mining, revealing the abundant mineral resources and rich deposits of coal and iron, and he utilized the Bulletin to access foreign investors. Finally, he collaborated with other partners who visited Spain and presented new members.

\section{Introducción}

La geología inició su constitución como ciencia propia e independiente a finales del siglo XVIII, una vez que se comenzaron a establecer unos términos referenciales -formaciones y sistemas- correctamente constituidos, enclasados y definidos (Álvarez, 2004). Entre 1810 y 1830 se produjo un salto cualitativo notable, creándose una comunidad geológica internacional y multiplicándose los intercambios y encuentros. En este contexto, la creación de un cuerpo doctrinal y la explicación de las distintas implicaciones de la causalidad histórico-espacial suscitaron intensas controversias entre los estudiosos de la materia, llegando en ocasiones "a tratarse mutuamente de un modo impropio entre hombres dedicados a ciencias" (Ezquerra, 1839). Pero las disputas entre unos y otros, en muchos casos más de orden filosófico que puramente geológico, no impidieron que se produjeran considerables avances. Así, tras 
González-Pumariega Solís, P. y González Prieto, L.A., 2017. Guillermo Schulz... Boletín Geológico y Minero, 128 (1): $221-240$

reconocerse la utilidad de los fósiles como elementos de referencia cronológicos, se sentaron las bases para progresar rápidamente en el establecimiento de los sistemas estratigráficos y se introdujeron los mapas geológicos con unidades litológicas, pasándose de la geognosia ortodoxa a la era de la estratigrafía paleontológica (Ellenberger, 1982). En este proceso resultaron determinantes las nuevas exigencias energéticas y productivas de hierro y carbón derivadas de la Revolución Industrial, ya que potenciaron una minería más activa que requería de un aparato teórico capaz de predecir dónde encontrar el mineral (Álvarez, 2000).

Francia trató de no permanecer ajena a este movimiento, pues acumulaba una larga tradición en el campo de las ciencias naturales. El Musée National $d^{\prime}$ Histoire Naturelle disponía de una cátedra de mineralogía desde su creación en 1793, y el Institut National des Sciences et des Arts, fundado dos años después y en el que se había integrado la prestigiosa Académie des Sciences, contaba también con una sección mineralógica. Además, tras la elección de la Convention nationale (1792) se había emprendido la organización estatal del ramo minero, por lo que la École des Mines, establecida en 1783 y reinstaurada en 1794, una vez superados los sucesos revolucionarios, habilitó su propia cátedra de mineralogía y geología.

Así, en 1811 y gracias al apoyo económico proporcionado por el Institut de France, el naturalista Georges Cuvier (1769-1832) y el ingeniero de minas Alexandre Brongniart (1770-1847) publicaron un trabajo puntero titulado Essai sur la géographie minéralogique des environs de Paris, en el que de forma precoz enumeraban las distintas formaciones geológicas de aquel entorno según su orden de superposición, su litología y sus fósiles. El texto se acompañaba de dos cortes y una carta geognóstica a escala 1:200.000, impresa en nueve colores planos, que se considera el primer mapa geológico francés moderno. Con estos productos cartográficos, Cuvier y Brongniart no solo mostraban a la comunidad científica el formidable poder de análisis que dichas herramientas proporcionaban a la nueva ciencia geognóstica, sino que también ilustraban y demostraban dos ideas completamente novedosas y revolucionarias para las teorías sobre la formación de la Tierra, como eran la alternancia de terrenos de sedimentación marina y continental, y la renovación de las faunas de una formación a otra (Ellenberger, 1982). Ese mismo año el profesor de geología y mineralogía de la École des Mines, André Brochant de Villiers (1772-1840) presentó al director general de minas un proyecto para la elaboración del mapa mineralógico de Francia, aunque la idea no prosperó en aquel momento.

Apenas cuatro años después (1815), el topógrafo británico William Smith (1769-1839) publicó su mapa de Inglaterra, Gales y parte de Escocia a escala de cinco millas por pulgada (equivalente a 1:316800) en quince hojas, siendo éste el primer mapa geológico de un país basado en el principio de la sucesión faunística, y en 1820 George Bellas Greenought (1778-1855) imprimió el Geological Map of England and Wales, realizado a escala de seis millas por pulgada (equivalente a 1:380.160) en seis hojas. Fue precisamente la publicación de éste último lo que motivó que en Francia se retomara la cuestión, acordándose realizar un mapa geológico del país a escala 1:500.000. La dirección de los trabajos recayó en Brochant, encomendándose la ejecución de los mismos a dos ingenieros de minas, Pierre-Armand Dufrénoy (1792-1857) y Léonce Élie de Beaumont (1798-1874). Los tres partieron en 1823 hacia Inglaterra con el fin de familiarizarse con las divisiones estratigráficas establecidas por los británicos. Una vez concluidos los trabajos de campo y de gabinete, la minuta del mapa fue presentada a la Académie des Sciences en 1835, realizándose la tirada definitiva en 1842 (Lacroix, 1928).

Mientras tanto, España se encontraba sumida en una profunda crisis social, política y económica que había llevado el cultivo de las ciencias a un estado crítico. Fue precisamente la pérdida de las posesiones americanas continentales y la gravedad de la crisis financiera lo que obligó al ministro de Hacienda durante la Década Ominosa, Luis López Ballesteros (1782-1853), a emprender una serie de importantes reformas, encontrándose entre ellas la de revitalizar la industria minera española. Para esta tarea recabó el apoyo de Fausto de Elhuyar (1755-1833), uno de los pocos científicos de altura que permaneció fiel al monarca absoluto. Gracias a él, en 1825 se creó la Dirección General de Minas y se promulgó el decreto-ley y la ordenanza que propiciarían el renacimiento de la minería peninsular-comenzando por la del plomo penibético- y regularían el desarrollo del ramo durante veinticuatro años. Además, con el fin de disponer de personal adecuadamente preparado con el que hacer frente a la estructuración de un cuerpo facultativo funcionarial, Elhuyar reabrió y dio nueva forma a la Academia de Minería de Almadén, pensionando en 1828 y 1829 a un grupo de cinco jóvenes a la prestigiosa Bergakademie de Freiberg (Sajonia). Asimismo, a finales de 1830 emprendió la ambiciosa tarea de formar el mapa geológico nacional, comisionando a Ángel Vallejo (1778-1840), un político liberal que aprovechaba su exilio en París adquiriendo conocimientos geológicos y mineralógicos en la 
École polytechnique y que había establecido relación con varios de los naturalistas más importantes del momento, para que iniciara el reconocimiento geognóstico de algunas provincias del norte peninsular (Aragonés, 1999).

\section{La creación de la Société Géologique de France}

A pesar de los avances experimentados en Francia, todavía existía entre los estudiosos de la geología de aquel país un cierto sentimiento de atraso respecto al grado de organización alcanzado por sus colegas británicos, que en 1807 habían logrado fundar en Londres la Geological Society. Esta institución, primera de su género en el mundo, publicaba desde 1811 las Transactions of the Geological Society of London y su primer director, Greenought, había emprendido en 1809 un importante proyecto de cooperación cartográfica en el seno de la asociación, que culminó una década después en el mapa de Inglaterra y Gales anteriormente citado.

En Francia, la Agence des mines de la République editaba desde 1794 el Journal des mines -denominado Annales des mines a partir de 1816-, que llegó a alcanzar una gran difusión y prestigio internacional. Más tarde, tras la supresión en 1823 del Journal de Physique, de Chimie, d'Histoire naturelle et des Arts, una de las publicaciones más antiguas en su género, los naturalistas Jean-Victor Audouin (1797-1841) y Jean-Baptiste-André Dumas (1777-1861), junto con el ingeniero de minas Adolphe Brongniart (18011876), imprimieron entre 1824 y 1833 los Annales des Sciences Naturelles. Asimismo, la Société pour la Propagation des Connaissances Scientifiques et Industrielles difundió entre 1824 y 1831 el Bulletin des Sciences Naturelles et de Géologie, a cargo de Ios naturalistas Gabriel Delafosse (1796-1878), Jean Baptiste Antoine Guillemin (1796-1842) y Jean Kuhn (1802-1868).

Sin embargo, a pesar de que estas publicaciones periódicas se vieron enriquecidas con numerosos trabajos geológicos y mineralógicos, en los años finales del primer tercio del siglo XIX no existía aún una revista francesa dedicada exclusivamente a la geología. Esta carencia fue la que llevó a los naturalistas Ami Boué (1794-1881) y Antoine-GabrielClaude Jobert (1797-1855) y al ingeniero geógrafo militar Claude-Antoine Rozet (1798-1858) a fundar en enero de 1830 el Journal de Géologie, pero sólo pudieron dar a la luz tres volúmenes antes de tener que cancelar su edición al año siguiente.

En este contexto, el influjo y la pujanza ejercida por la Geological Society of London había llevado a un reducido grupo de naturalistas residentes en París a plantearse en 1828 la posibilidad de crear una asociación similar. Louis-Constant Prévost (1787-1856), discípulo de Brongniart aunque opuesto a la teoría catastrofista, fue quien llevó el peso de este proyecto. Tras consultar con algunos amigos, redactó un par de artículos de incorporación, pero fue la aparición de la revista Journal de Géologie lo que aceleró la ejecución del plan.

Así, a comienzos del mes de marzo de 1830 Prévost se reunió en la oficina de Boué con Louis Cordier (17771861), Henri Marie Ducrotay de Blainville (1777-1850), el barón de Férussac (1786-1836), Félix de Roissy (1771-1843) y Jobert, acordando constituir la sociedad geológica. Curiosamente, ni Brochant, que en aquel momento dirigía las operaciones para la elaboración del mapa geológico del país, ni Brongniart, fueron invitados a las conversaciones, aunque ambos se alegraron de la novedad. El día 17 de ese mismo mes lograron congregar a un grupo de cuarenta personas interesadas en el progreso de la geología y, tras algunas deliberaciones, adoptaron las bases definitivas de la asociación (Boué, 1861). Con su establecimiento, la Société Géologique de France se proponía como objetivo general contribuir al avance de la geología $y$, de forma más concreta, dar a conocer el suelo del territorio nacional, «tanto en sí mismo como en sus relaciones con las artes industriales y la agricultura».

En la segunda sesión, celebrada el 28 de mayo, se constituyó la primera junta directiva, y en la tercera -21 de junio- se designó a los componentes del consejo administrativo. El 25 de agosto la asociación fue presentada al rey Louis-Philippe, recién llegado al trono tras la revolución de julio. En el acto de recepción, Prévost centró su intervención en la necesaria libertad de acción y pensamiento de sus miembros - «señor, para llegar a florecer, las ciencias necesitan libertad»-. Con ésta demanda ponía de manifiesto el afán de emanciparse de las directrices marcadas por la Académie des Sciences, cuyo secretario general perpetuo-Cuvier- mantenía unos postulados fixistas que impedían el desarrollo de nuevos puntos de vista científicos, como las ideas transformistas de JeanBaptiste de Lamarck (1744-1829) (Laurent, 2006). Por orden del rey, el 3 de abril de 1832 la asociación logró su reconocimiento como institución de interés público.

Fiel a su propósito fundacional, la Société Géologique de France se convirtió rápidamente en un elemento esencial para el desarrollo de la geología. Durante las sesiones se llevaban a cabo muchos debates que permitían a sus miembros mantenerse al tanto de los avances científicos e intercambiar ideas. Asimismo, se organizaban reuniones extraordinarias 
anuales en diferentes localidades que congregaban a un número significativo de geólogos. Además, desde su creación la asociación contó con un órgano de difusión particularmente eficaz, el Bulletin de la Société Géologique de France -en adelante BSG-, que con carácter mensual y una tirada ordinaria de quinientos ejemplares, era remitido de forma gratuita a todos sus socios (Fig. 1). Gracias a él, los miembros que no residían en París podían seguir los debates que tenían lugar en las sesiones y ponerse al día de los acontecimientos. También era de gran utilidad para que los geólogos locales, profesionales o aficionados, pudieran dar a conocer los resultados de sus trabajos. No obstante, y dado que era una revista de pequeño tamaño -en octavo- que contenía muy pocas ilustraciones, en 1833 se comenzaron a editar las Mémoires de la Société Géologique de France con el fin de dar cabida a grandes monografías, siendo ésta ya una publicación de gran formato -en cuarto-ilustrada con numerosas planchas y mapas, a menudo coloreados, que los socios podían adquirir por un precio especial.

Para la admisión de nuevos asociados se fijó un derecho de entrada de 20 francos y una suscripción anual de 30 , pudiéndose incorporar cualquier extranjero interesado en igualdad de condiciones. En

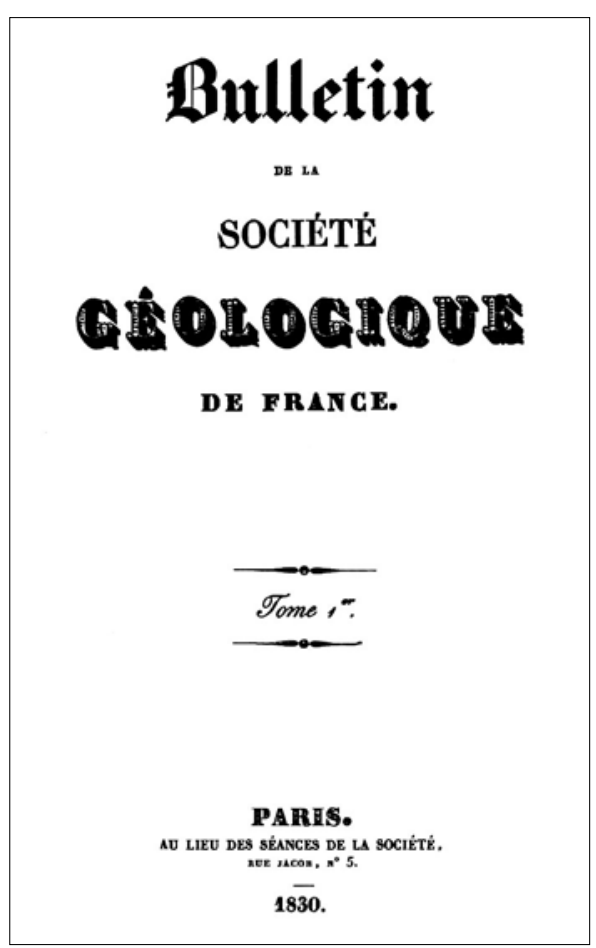

Figura 1. Portada del primer número del Bulletin de la Société Géologique de France (1830).

Figure 1. Cover of the first issue of the Bulletin of the Geological Society of France (1830). noviembre de 1830 la sociedad contaba ya con 143 miembros, de los que 30 eran extranjeros (21\%); en 1833 eran 221 y 64 (29\%), respectivamente; en 1836, 302 y $97(32 \%)$ y dos décadas después de su fundación -1850- agrupaba a 513 socios, con 186 (36\%) residentes fuera del país. Estas cifras ponen de manifiesto que el trabajo de los miembros de la Société Géologique de France fue ampliamente reconocido en el extranjero (Laurent, 2006).

\section{Guillermo Schulz y su incorporación a la Dirección General de Minas de España}

Guillermo Schulz nació el 6 de marzo de 1805 en la parroquia de Dörnberg (Principado electoral de Hesse-Kassel). Su abuelo había sido minero en la localidad de Blankenbach y su padre era el responsable-Obergeschworner- de la vecina mina de lignito sita en Habichtswald. En 1823 uno de sus hermanos se incorporó a esta explotación como capataz subalterno-Grubensteiger-. Sin embargo, al cumplir los 18 años él optó por matricularse en la prestigiosa GeorgAugust-Universität Göttingen para seguir los cursos de metalurgia -studiosus rei metallicae- (Truyols y Schroeder, 2005). En ella impartía docencia el eminente mineralogista Johann Friedrich Ludwig Hausmann (1782-1859), con el que el joven alumno estableció una estrecha relación que mantendría de por vida.

Tras completar su formación teórica con prácticas en las minas y fundiciones del Harz, Turingia y Sajonia, a finales de 1825 Schulz fue requerido por una compañía anglo-española para dirigir las obras de exploración y laboreo de sus minas de plomo en la sierra de Gádor y comarcas adyacentes (Maffei, 1877). Esta empresa había construido en la localidad de Adra una de las más avanzadas instalaciones metalúrgicas de la época, dotada en 1824 con seis hornos ingleses alimentados por carbón de piedra y tres años después con una máquina a vapor (Madoz, 1845), y pretendía acometer la excavación de un socavón general de registro que permitiera aplicar los modernos métodos de extracción para lograr el aprovechamiento organizado de sus criaderos metálicos. De esta forma, Schulz llegó a España en marzo de 1826, desempeñando durante cuatro años dicho encargo particular a plena satisfacción de sus patrones, aunque las cortapisas establecidas por la legislación minera para evitar la acumulación de los recursos en pocas manos hicieron fracasar finalmente el ambicioso proyecto (Maestre, 1845).

Poco antes de finalizar su compromiso laboral, en la primavera de 1829 recibió la visita de Hausmann, que había sido enviado por las autoridades de su país 
para determinar si la inesperada y cuantiosa producción penibética de plomo podía representar una amenaza para los mercados y las minas europeas. Así, pudo proporcionarle de primera mano datos sobre este asunto y también otros de especial interés para la descripción geognóstica de España que su maestro redactó dos años después (Hausmann, 1831).

Concluido su contrato, a finales de 1829 Schulz visitó los establecimientos mineros de Linares y Almadén, tras lo cual regresó a la Confederación Germánica, pasando por Madrid y París, para recorrer seguidamente diversas instalaciones minero-metalúrgicas de Sajonia, Bohemia, Silesia y Hungría con el fin de adquirir nuevos conocimientos (Maffei, 1877). Durante ésta primera etapa en España, el ya curtido ingeniero se dio a conocer en un país que no había participado activamente en el nacimiento de la nueva ciencia geológica y cuya práctica se acometería con un cierto retraso respecto a las principales naciones europeas (Fig. 2).

En este estado de cosas, el 24 de julio de 1830 Schulz solicitó desde Praga una de las plazas de comisario de minas que aún se encontraban vacantes en la Dirección General. Todo parece indicar que, tras su partida de Andalucía, se debió reunir en Madrid con Elhuyar para informarle del estado de la industria minera-metalúrgica alpujarreña y, a su vez, éste le debió manifestar la necesidad que tenía de contar con algún ingeniero experimentado para cubrir el cargo de comisario previsto en el organigrama funcionarial. El nombramiento por el que Schulz quedó vinculado a la administración española se produjo el 29 de septiembre, aunque se le autorizó para que durante un año concluyera su periplo formativo. Así, desde Praga pasó a Viena, continuando por Estiria, Baviera, los países del Rin, Suiza y finalmente Francia, donde permaneció en París entre el 2 y el 20 de octubre de 1831.

Allí tuvo ocasión de establecer contacto con algunos destacados miembros de la recién creada Société Géologique de France, como Cordier y Brongniart, presidente y vicepresidente de la misma. Además, Boué, secretario de la asociación, le enseñó junto con Charles-Marie D'Orbigny (1770-1856) un boceto de la carta geológica del país realizada por Dufrénoy y Beaumont bajo la dirección de Brochant de Villiers, y le dio sendos ejemplares del segundo y tercer tomo del Journal de Géologie en los que se habían publicado, respectivamente, las memorias del ingeniero de caminos Antonio Gutiérrez (i?-1840) sobre los terremotos de Murcia y del coronel Charles Silvertop (1781-1839) sobre las cuencas de Baza y Alhama (Granada) (González-Pumariega, 2011). En justa correspondencia, el germano se comprometió a remitirles noticias geológicas sobre España (Boué, 1832).

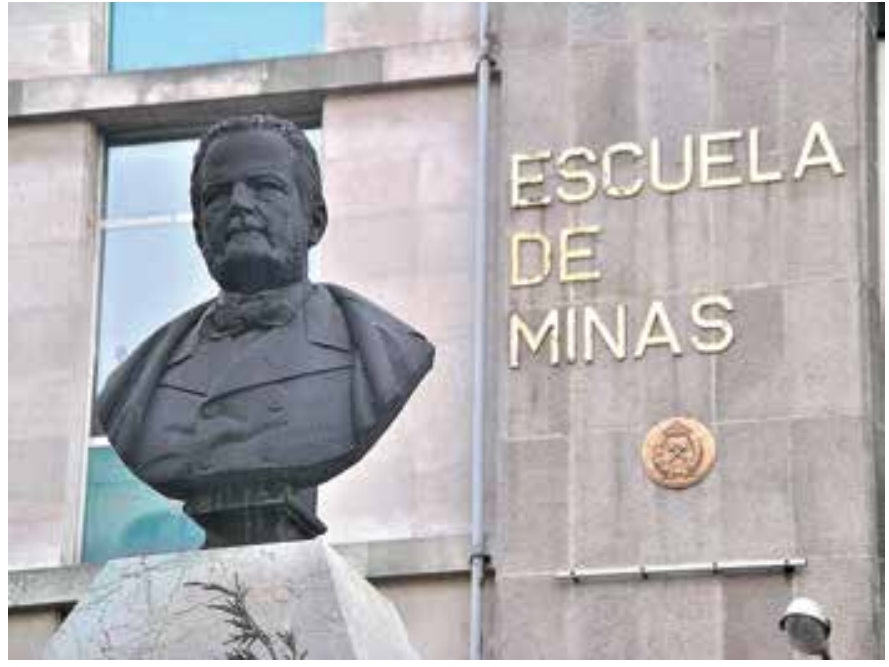

Figura 2. Busto de Guillermo Schulz en la Escuela de Minas de la Universidad de Oviedo.

Figure 2. Bust of William Schulz at the School of Mines (University of Oviedo).

Una vez finalizada su comisión instructiva, Schulz llegó a Madrid a finales de 1831, partiendo en el mes de diciembre para Galicia, su primer destino facultativo.

\section{Aportaciones científicas de Schulz a la Société Géologique de France}

\section{La Descripción geognóstica del Reino de Galicia}

Dada la lentitud con la que Vallejo estaba desarroIlando la comisión de comenzar a elaborar el mapa geológico nacional por las provincias del norte de la Península, en 1832 la Dirección General de Minas decidió impulsar dicho proyecto encomendando a Schulz la realización de una descripción geognóstica y un mapa petrográfico de Galicia (R.O. del 27 de mayo).

Para poder abordar este encargo, el germano tuvo que superar las dificultades que su apartado destino le imponía para mantenerse al día de los incesantes progresos experimentados por la geología, logrando hacerse con las últimas obras publicadas en su facultad. Asimismo, solicitó el ingreso en la Société Géologique de France, en cuyo seno fue admitido en la sesión celebrada el 18 de marzo de 1833, siendo presentado por el secretario en funciones Jules Desnoyers (1800-1887) y por Boué, miembro del consejo (Fig. 3). En aquel momento, solo tres españoles formaban parte de la asociación: Vallejo, el recién fallecido Elhuyar y el sustituto de éste al frente de 
González-Pumariega Solís, P. y González Prieto, L.A., 2017. Guillermo Schulz... Boletín Geológico y Minero, 128 (1): $221-240$

\section{Séance du 18 mars 1833.}

Présidence de M. de Bonnard.

Après la lecture et l'adoption du procès-rerbal de la dernière séance, $M$. le président proclame membres de la Société.

\section{MM.}

Schurz (G.), employé supérieur des mines à Lugo, en Espagne, présenté par MM. Boué et Desnoyers;

Figura 3. Nombramiento de Schulz como miembro de la Société Géologique de France (BSG, Tome III, 1er. série, p. 211).

Figure 3. Schulz's appointment as a member of the Geological Society of France.

la Dirección general de minas, Timoteo Álvarez de Veriña (1739-1834) (Viquesnel, 1844).

No ha quedado constancia de que Schulz enviara a la sociedad las noticias geológicas sobre España que había ofrecido durante su visita a París en 1831, pero antes de concluir su Descripción Geognóstica del Reino de Galicia, el 30 de mayo de 1834 remitió, como primicia, un resumen de la memoria en el que sintetizaba las principales características de la región. En él manifestaba que no había concluido aún sus investigaciones sobre ciertos materiales margosos, debido a la ausencia de fósiles, por lo que los había considerado inicialmente como depósitos terciarios. La sociedad dio cumplida cuenta de este informe en la sesión celebrada el 7 de julio de 1834 (BSG, Tomo IV, $1^{\text {a }}$ serie, pp. 416 a 418) (Fig. 4). Además, Boué mencionó la conclusión de los trabajos de campo de Schulz

416 SEANGE DU 7 JUILIET 1834.

M. Bidard écrit à la Société pour lui adresser une note sur la carbonisation du bois. La lecture de cette notice est renvoyée à l'une des prochaines séances.

M. Paul Savi annoncel'envoi de sept volumes, formant la continuation des actes de l'Académie des géorgofiles de Florence.

M. Guillaume Schulz, inspecteur des mines des Asturies et de la Galice, adresse la lettre suivante, datée du 3o mai, de Rivadeo, en Galice.

J'ai presque achevé un relevé et une description géologrique de la Galice, savoir, quinze feuilles de manuscrit et une carte géologique d'un pied carré. Voici les faits principaux, en attendant que j'adresse à la Société tout mon manuscrit.

Figura 4. Presentación del avance de la Descripción geognóstica de Galicia (BSG, Tome IV, 1er. série, p. 416).

Figure 4. Presentation of the summary of the Geognostic description of Galicia. en su Résumé des progrés des Sciences Géologiques en 1833 ( $B S G$, Tomo V, $1^{\text {a }}$ serie, p. 505) y el contenido de su informe fue extractado por Rozet en el Resume des travaux de la Société Géologique de France, et des progrès de la géologie en France, depuis le 1er novembre 1833, jusqu'au $7^{\text {er }}$ novembre 1834 (BSG, Tomo VI, $1^{\text {a }}$ serie, pp. LII y LIII).

Finalmente, y pese a tener que simultanear estas labores científicas con sus deberes oficiales, Schulz entregó su trabajo, junto con una colección de 184 muestras de rocas, en el perentorio plazo de dos años que se le había fijado, siendo publicado de real orden a principios de 1835. En la introducción de su opera prima, manifestaba que la geognosia debía tener como fin primordial dar a conocer a la sociedad las ventajas que ésta podía obtener del conocimiento de la constitución de la superficie terrestre. Sin embargo, reconocía que, dado el tiempo y los medios disponibles, su labor se había limitado a proporcionar un conocimiento general de aquello que la región podía ofrecer por la calidad de su suelo, enumerando todas las rocas que se hallaban con alguna extensión, clasificándolas geognósticamente, indicando los usos y la aplicación que tenían o podían tener, y formando "más bien un croquis petrográfico de Galicia que un mapa perfecto». Otra dificultad añadida indudable, pero que evitó mencionar, fue que el año anterior el gobierno había incluido a la geología entre las materias a las que se imponían una serie de restricciones respecto a su divulgación y desarrollo con el fin de asegurar que no contuvieran ideas contrarias a la religión (R.D. del 4 de enero de 1834).

Dado el estado naciente de la ciencia geológica en aquel momento, Schulz no acertó a comprender algunas formaciones y cometió errores puntuales al identificar ciertos materiales o la génesis de algún terreno (Vidal, 2005). No obstante, su trabajo destaca por la gran cantidad de información que logró reunir, lo acertado de la mayoría de sus interpretaciones y sus numerosos descubrimientos, constituyendo el primer bosquejo geológico publicado sobre una parte considerable del territorio de la Península (Fernández de Castro, 1874). La memoria incluye, además, una Tabla de algunos términos geognósticos usuales en la minería, en la que registró 37 entradas para designar terrenos en masa, 66 para terrenos estratificados $y$ 23 términos de minería en alemán, francés y español. Esta relación supuso una novedad en nuestro idioma, en el que aún no estaban bien fijados muchos de ellos, y aunque refleja todavía la influencia de la clasificación werneriana, Schulz empleó sobre el terreno unidades menores descriptivas, similares o idénticas a las propuestas por Smith, lo que evidencia que sus 
métodos de trabajo eran empiristas $y$, por lo tanto, modernos para la época (Álvarez, 2004).

Una vez entregados a la imprenta, el 10 de enero de 1835 notificó a la sociedad que en breve remitiría su libro y mapa sobre Galicia. También aprovechó la ocasión para indicar que en aquel momento estaba trabajando con Lorenzo Gómez Pardo (1801-1847) en la reforma de la legislación minera, pero que el gobierno le había encargado realizar un nuevo trabajo geognóstico sobre Asturias ( $B S G$, Tomo $\mathrm{V}, 1^{\text {a }}$ serie, sesión del 26 de enero de 1835, p. 125. Ese mismo día se informó de que Vallejo había abandonado momentáneamente la geología «al recibir varios encargos del gobierno relacionados con la educación pública»). La donación de dos ejemplares de la Descripción Geognóstica del Reino de Galicia fue recibida el 29 de junio ( $B S G$, Tomo V, $1^{\text {a }}$ serie, p. 341). En una nota adjunta al envío, Schulz explicaba que tras haber comparado los materiales dudosos con otros similares de Castilla, los consideraba ya como indudablemente pertenecientes a la formación de Keuper -terreno que había sido definido el año anterior por Friedrich August von Alberti (1795-1878) (GarcíaCortés, 2005)-, por lo que los había clasificado como margas arenosas y margas irisadas pertenecientes al terreno secundario.

\section{La descripción geológica de Asturias, con un atlas}

Coincidiendo con el final del régimen absolutista y la instauración de la regencia de $\mathrm{M}^{\mathrm{a}}$ Cristina de Borbón, en septiembre de 1833 se creó el Real Cuerpo Facultativo de Minas. A resultas de ello, Schulz fue nombrado inspector del distrito de Asturias y Galicia.

Cuatro años antes, el empeño del gobierno por promover el aprovechamiento del carbón de piedra había llevado a nombrar una comisión compuesta por los facultativos Joaquín Ezquerra del Bayo (17931859), Francisco de Sales García (i?-i?), Rafael Amar de la Torre (1802-1874) y Felipe Bauzá Rábara (1801$1875)$, quienes a comienzos de 1830 practicaron el reconocimiento geológico de los abundantes yacimientos hulleros asturianos y propusieron las mejores vías para su transporte. Asimismo, se establecieron conversaciones con el industrial John Cockerill (17901840), radicado en Lieja, con la intención de establecer una gran fundición siderúrgica en la provincia, pero el intento no prosperó. Sin embargo, gracias a la concesión de unos privilegios especiales, a finales de 1833 el financiero valón Nicolas-Maximilien Lesoinne (1774-1839) constituyó, junto con su hijo e ingeniero de minas Philippe-Adolphe Lesoinne (1803-1865) y los capitalistas españoles exiliados Joaquín $\mathrm{M}^{\mathrm{a}}$
Ferrer (1777-1871) y Felipe Riera (1790-1881), la Real Compañía Asturiana de Minas de Carbón con el fin de explotar tres minas en la comarca de Avilés.

Uno de los principales cometidos del inspector de distrito sería el de auxiliar a ésta y otras empresas que se pudieran establecer en su demarcación, si bien los continuos vaivenes políticos y las severas dificultades económicas que padeció el país, junto con las gravosas contribuciones que la ley exigía a los industriosos, lastraron aún durante algunos años el progreso de la minería asturiana. Pero además de atender a sus deberes facultativos, Schulz tuvo que continuar desarrollando una labor científica fundamental, ya que, tal como había indicado a la Société Géologique de France, apenas hubo concluido su trabajo sobre Galicia le fue encomendada la comisión extraordinaria de componer la descripción geognóstica y el mapa petrográfico de Asturias en dos años (R.O. del 4 de diciembre de 1834).

No obstante, una serie de circunstancias adversas determinaron que la ejecución de este encargo se viera interrumpida en varias ocasiones, dilatándose finalmente durante dos décadas. A pesar de ello, a mediados de 1837 ya se había formado una idea aproximada de la conformación geológica general de la provincia, caracterizada por una mayor diversidad de terrenos que Galicia pero, sobre todo, por el complejo enlace que estos presentaban entre sí. Sin pérdida de tiempo, preparó un pequeño artículo describiendo las diversas clases de terreno presentes en la región y lo remitió a la sociedad con el título D'une notice abrégée géognostique sur les Asturies (Vitar, 2010). Su contenido fue presentado por el miembro del consejo Émile Le Puillon de Boblaye (1792-1843) en la sesión celebrada el 3 de septiembre ( $B S G$, Tomo VIII, $1^{\text {a }}$ serie, pp. 325 a 328) (Fig. 5). En él Schulz seguía dando muestras de adoptar la sistemática más reciente al emplear los términos Système Cambrien y Système Silurien, definidos respectivamente por Adam Sedgwick (1785-1873) y Roderick Murchison (1792-1871) en 1835 al haberlos reconocido en los terrenos de transición del país de Gales (Puche y AyalaCarcedo, 2001), siendo comunicadas estas observaciones a la sociedad por Beaumont en la sesión del 11 de enero siguiente, tras identificarlos, junto con Dufrénoy, con la misma disposición y divisiones en la Bretaña francesa ( $B S G$, Tomo VII, $1^{\text {a }}$ serie, pp. 90 y 91).

En 1838 la Notice apareció, ligeramente ampliada y con el título de Reseña Geognóstica del Principado de Asturias, en el primer volumen de los Anales de Minas (pp. 361 a 378), publicación con la que, remedando a la homónima francesa, la Dirección General de Minas pretendía contribuir al desarrollo del ramo. 


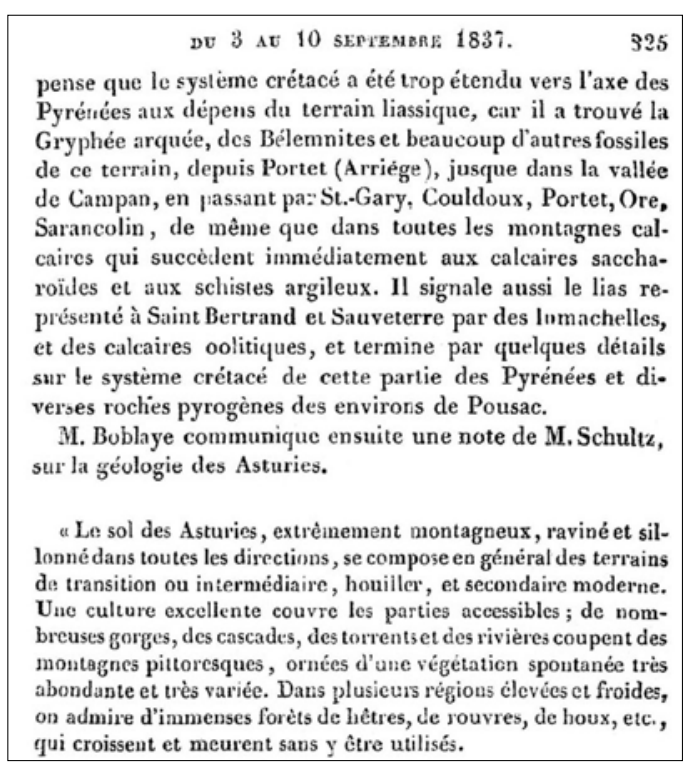

Figura 5. Presentación de las Breves noticias geognósticas sobre Asturias (BSG, Tome VIII, 1er. série, p. 325).

Figure 5. Presentation of the Brief Geognostic News about Asturias.

A manera de preliminar, Schulz añadió una descripción orográfica de la provincia, en la que destacaba la peculiaridad geomorfológica de la rasa costera, aunque sin alcanzar a comprender su origen. Además, incorporó detalles adicionales sobre cada uno de los tipos de terreno y amplió la relación de fósiles encontrados. Como indicaciones especialmente relevantes, señalaba que la pudinga y el conglomerado del Carbonífero requerían una observación más rigurosa, dada su peculiaridad; manifestaba sus dudas sobre la presencia de la "formación juránea» en la región y consideraba que alguna porción del terreno de acarreo antiguo podría ser terciario, aunque suspendía su clasificación definitiva por no haber encontrado fósiles. Aparte de estos aspectos «instructivos para la geología», también prestó una mayor atención a los minerales útiles, pensando en su posible aprovechamiento minero y fabril. Esta intención se debe relacionar con su esperanza de que se produjera un pronto amanecer industrial en la región tras la conclusión de la guerra civil, que resulta aún más explícita en su Ojeada sobre el estado actual de la minería del distrito de Asturias y Galicia, publicada en el mismo tomo de los Anales (pp. 379 a 397). Fiel a su compromiso de colaboración con la Société Géologique de France, Schulz remitió sendos ejemplares de la Reseña y la Ojeada, que había impreso como separatas, dándose cuenta de dicha donación en la sesión del 3 de junio de 1839 (BSG, Tomo $\mathrm{X}, 1^{\mathrm{a}}$ serie, pp. 319 y 320 ).
Las expectativas del inspector del distrito no eran infundadas, puesto que a comienzos de 1838 el financiero español afincado en París, Alejandro Aguado (1784-1842), marqués de las Marismas del Guadalquivir, había constituido una serie de sociedades a nombre de varios testaferros con la intención de sortear las limitaciones que la legislación de minas establecía para llegar a componer un gran coto minero (González, 2015). Esta artimaña legal fue consentida por la administración del ramo, ya que dejaba «la puerta abierta para que la industria minera esté al alcance de los pequeños capitales y no sea una exclusiva y un monopolio de los potentados" (Ezquerra, 1844). Así, en los meses de abril y junio un representante de Aguado registró a nombre de varias empresas gran número de pertenencias mineras sobre los mejores criaderos de hulla de los concejos de Langreo y Siero, contando para ello con las indicaciones proporcionadas por Schulz (Lobé, 1842). Una vez obtenidas las concesiones, en noviembre Aguado envió a Asturias a los ingenieros de minas NicolasArmand Buvignier (1808-1880) y François-Clément Sauvage (1814-1872) para que llevaran a cabo un reconocimiento facultativo de los yacimientos, a raíz del cual elaboraron un informe titulado Rapport sur les mines de Siero et Langreo, que no fue publicado.

Enterado de la visita de sus colegas franceses, Schulz quiso aprovechar la magnífica ocasión que se le presentaba para compartir con ellos sus experiencias y conocimientos, preparándoles un itinerario de interés geológico-minero por la provincia, aunque limitado a la franja septentrional debido a los rigores invernales. Una carta remitida al contador de la Real Compañía Asturiana de Minas el 15 de octubre permite comprobar el interés que puso en el asunto, al advertirle de una "próxima y acaso larga ausencia de Ribadeo", localidad en la que se encontraba la sede de la inspección del distrito (Archivo histórico de Asturiana de Zinc, S.A.).

Tras su regreso a Francia, Buvignier, miembro de la sociedad geológica desde 1835 , recogió sus impresiones científicas en un opúsculo titulado Note géologique sur les Asturies, que comunicó a la asociación en la sesión que tuvo lugar el 4 de febrero de 1839 (BSG, Tomo X, $1^{\text {a }}$ serie, pp. 100 a 104). Este artículo aporta varios detalles interesantes, pero destaca especialmente por los cinco cortes geológicos esquemáticos que complementan el texto y con los que el autor pretendía mostrar la posición relativa de los diferentes terrenos observados -aparecen insertos en una lámina aparte junto con los de otros colaboradores (figs. 4 a 8, p. 78)-.

Aunque ya la comisión encargada del estudio de los criaderos de carbón de piedra había dibujado en 
su momento cuatro secciones geognósticas interpretativas (Ezquerra et al., 1831), su ámbito de trabajo estuvo limitado a la zona central de Asturias, por lo que los cortes publicados en el Bulletin son los primeros que comprenden todas las formaciones reconocidas por aquel entonces en la provincia, si bien con la simplicidad característica de la época en lo que se refiere a la tectónica. En todo caso, y puesto que no habían podido penetrar hacia el sur del Principado, Buvignier reconoció que dichos bocetos eran, en mayor medida, "resultado de mis conversaciones con el Sr. Schulz, más que de mis propias observaciones", por lo que en realidad sintetizan lo expuesto por éste en sus dos escritos anteriores.
De forma muy elemental, los cuatro primeros cortes muestran la disposición de los diferentes tipos de terreno según una sección norte-sur, estando trazados, respectivamente, a la altura de Colombres (fig. 4), Ribadesella (fig. 5), Villaviciosa (fig. 6) y Soto de Luiña (fig. 7), mientras que el quinto (fig. 8), más generalizado aún, configura una especie de arco en sentido este-oeste entre Colombres (Ribadedeva) y La Vega (Vegadeo). Como curiosidad, cabe señalar que la terminología empleada por Buvignier no está tan actualizada como la de Schulz, puesto que recurre a la designación de terrain ardoisier y terrain anthraxifère adoptada treinta años antes por JeanBaptiste Julien d'Omalius d'Halloy (1783-1875) para
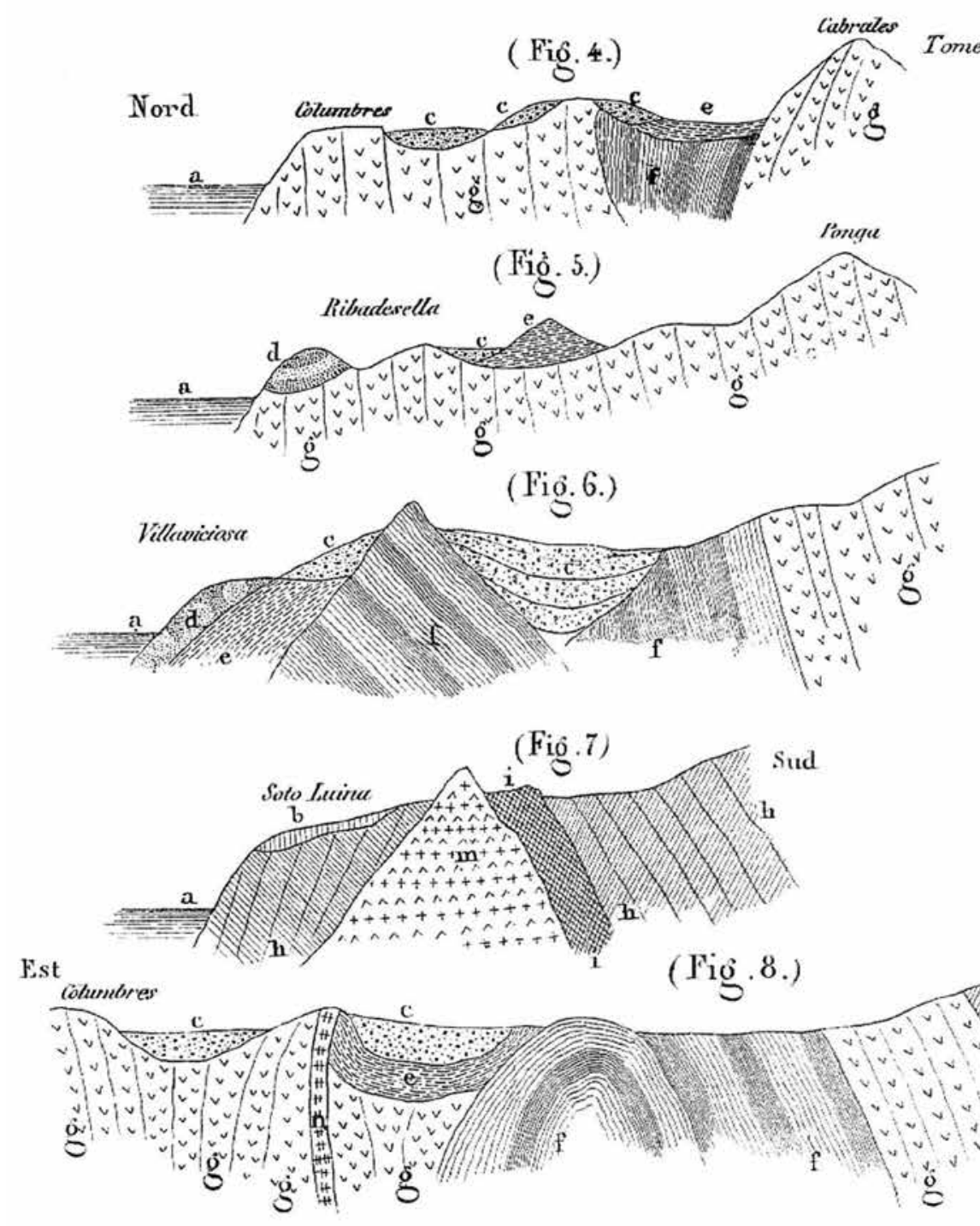

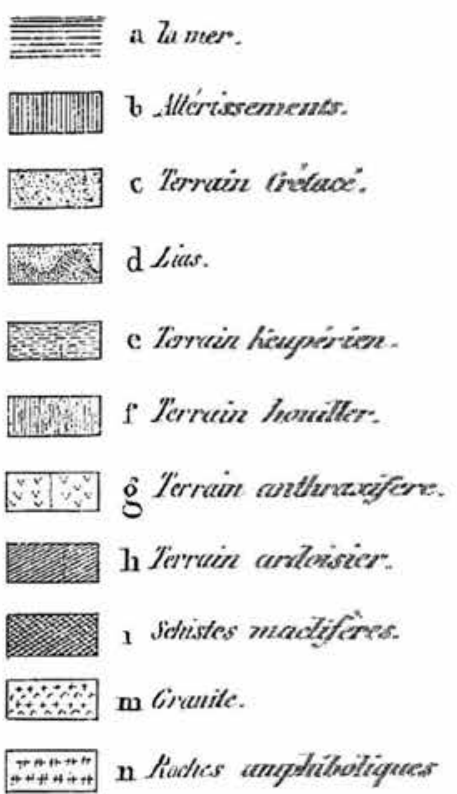

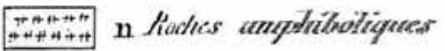

Figura 6. Cortes que acompañan a la Nota geológica sobre Asturias (BSG, Tome X, 1er. série, p. 78).

Figure 6. Cross sections accompanying the report on the geology of Asturias. 
González-Pumariega Solís, P. y González Prieto, L.A., 2017. Guillermo Schulz... Boletín Geológico y Minero, 128 (1): $221-240$

referirse a los wernerianos terrenos de transición Übergangsgebirge- (Fig. 6).

La Königliche Gesellschaft der Wissenschaften zu Göttingen -Real Sociedad de Ciencias de Gotingadio cuenta de la publicación del artículo de Buvignier en el primer volumen del año 1840 del Göttingische gelehrte Anzeigen, destacando el papel desempeñado por Schulz, y al considerar poco probable que sus últimos escritos se hubieran difundido en Alemania, también aprovechó la ocasión para reproducir íntegramente su Reseña geognóstica de la provincia de Asturias y resumir el contenido de la Ojeada sobre el estado actual de la minería en el distrito de Asturias y Galicia.

Entretanto, en aquel momento se crearon, además, otras dos compañías importantes para la explotación del carbón de piedra en las localidades de Santofirme y Ferroñes (Llanera). Entre todas solicitaron en apenas dos años hasta 252 pertenencias mineras, lo que recargó a Schulz de trabajo burocrático. Este se incrementaría aún más en 1841, cuando, muy a su pesar, fue trasladado a Madrid como vocal de la Dirección General de Minas. Allí logró mantenerse al margen de las disputas políticas que se suscitaron en el seno de la administración minera, por lo que se libró de las consiguientes depuraciones habidas tras la alternancia de gobiernos progresistas y moderados. De esta forma fue ascendiendo en el escalafón del cuerpo de ingenieros de minas, hasta alcanzar en 1844 el cargo de inspector general primero. Pero sus obligaciones administrativas le impidieron mantener la necesaria regularidad en el estudio de la geología de Asturias, viéndose obligado a hacerlo por temporadas, lo que demoró su conclusión.

No obstante, a lo largo de este tiempo publicó diversos trabajos, remitiendo los más significativos a la sociedad. De esta forma, el 4 de abril de 1842 se recibió la Estadística de la minería de Asturias y Galicia (BSG, Tomo XIII, $1^{\text {a }}$ serie, p. 256 y 539 ); el 3 de febrero y el 17 de marzo de 1845 todos los números del Boletín Oficial de Minas correspondientes al año 1844 y el de enero de 1845, en los que había insertado, en varias entregas, sus Breves informes sobre el estado actual de algunas minas de carbón de Asturias y la Reseña de los principales criaderos de carbón en Asturias, con los que pretendía atraer la atención de posibles inversores, además de los artículos titulados Relación calificada de las minas de Vizcaya que se hallaban en labor en Noviembre de 1844 y Breve reseña de las minas de la provincia de Santander (BSG, Tomo II, $2^{\text {a }}$ serie, pp. 257 y 327); el 1 de diciembre de 1845 se dio cuenta del envío de una segunda edición de la Reseña geognóstica del Principado de Asturias, en la que incluía algunas anotaciones para explicar que la mayor parte del terreno que aparecía descrito como "Siluriano" correspondía en realidad al "Devoniano", división que aún no se había establecido cuando la redactó por primera vez, e informaba del descubrimiento de nuevos criaderos de galena argentífera y de cinabrio, así como de "huesos de animales antediluvianos" en varias cavernas (BSG, Tomo III, $2^{\text {a }}$ serie, p. 60 y 788); por último, en 1848 se dio noticia de la publicación de su Vistazo geológico sobre Cantabria (BSG, Tomo V, $2^{\mathrm{a}}$ serie, p. 635).

La Société Géologique de France también tuvo conocimiento de los trabajos de Schulz en Asturias gracias a los artículos publicados en el Bulletin por algunos otros miembros. El más prolífico en este sentido fue Antoine-Adrien Paillette (1809-1858), ingeniero de minas francés que llegó a la región a comienzos de la década de 1840 para dirigir las explotaciones de Ferroñes y permaneció en ella hasta 1857, llevando a cabo una meritoria labor industrial. Durante los primeros años practicó además numerosos reconocimientos geológicos y mineralógicos, así como una gran cantidad de análisis químicos para diversas compañías y particulares, lo que le permitió suscribir varios informes y monografías que remitió a la sociedad (Llaneza, 2007). En casi todos ellos, Paillette citó elogiosamente a Schulz y reconoció su autoridad científica, proporcionando igualmente algún dato sobre sus progresos. De esta forma, en la sesión del 20 de abril de 1846 se leyó una carta suya en la que decía: "todos esperamos ansiosamente aquí el mapa de nuestro distinguido colega D. Guillermo Schulz" (BSG, Tomo III, $2^{\text {a }}$ serie, pp. 450 a 454), y tres años después -sesión del 18 de junio de 1849- daba a entender que el germano ya había dibujado el mapa geográfico y geológico de Asturias, indicando que era tan sobresaliente por la precisión topográfica como por la que había puesto en el trazado y la delimitación de los terrenos (BSG, Tomo VI, $2^{\text {a }}$ serie, pp. 575 a 599).

En su afán investigador, Paillette animó a los paleontólogos Édouard de Verneuil (1805-1873) y Adolphe d'Archiac (1802-1868) a que visitaran Asturias en el verano de 1844, haciendo en su compañía un recorrido por la provincia recogiendo fósiles. En los meses de mayo y junio Schulz también estuvo en la región, por lo que seguramente haría todo lo posible para coincidir con ellos. En todo caso y como reconocimiento a su labor, los naturalistas franceses le darían al año siguiente el nombre de Pentremites schulzii (=Oryptoschisma schulzii) a una nueva especie de blastoideo que identificaron en el terreno paleozoico de Ferroñes ( $B S G$, Tomo II, $2^{\text {a }}$ serie, pp. 479 y 480). Además, en 1850 Verneuil denominó Terebratula schulzii (=Cimicinella schulzii) a un braquiópodo que había localizado en el Devónico de 
Sabero (León) (BSG, Tomo VII, $2^{a}$ serie, p. 161) y en 1855 Verneuil y Joachim Barrande (1799-1883) bautizaron como Avicula schulzii a un bivalvo encontrado en el terreno devoniano de la comarca de Almadén (Ciudad Real) (BSG, Tomo XII, $2^{\mathrm{a}}$ serie, pp. 964 a 1025) (Fig. 7).

Por otra parte, en la sesión del 6 de diciembre de 1852 Verneuil y Édouard Collomb (1801-1875) avisaron a los miembros de la sociedad de que Schulz estaba terminando ya el excelente mapa geográfico y geológico de Asturias «que le había costado más de quince años de trabajo y que la ciencia esperaba con impaciencia" (BSG, Tomo X, $2^{\text {a }}$ serie, pp. 133 y 139). Sin embargo, la impresión del Mapa Topográfico de la Provincia de Oviedo se demoró hasta 1855, notificándose su donación a la sociedad en la sesión del 19 de noviembre (BSG, Tomo XIII, $2^{\text {a }}$ serie, p. 58), y su obra magna, la Descripción Geológica de Asturias, con un Atlas, no vio finalmente la luz hasta 1858, dándose cuenta de su recepción en la asociación en la sesión del 16 de mayo del siguiente año ( $B S G$, Tomo $\mathrm{XVI}, 2^{\mathrm{a}}$ serie, p. 667).

El texto comenzaba con una reseña preliminar, articulándose el resto en nueve secciones principales, dedicadas a cada uno de los terrenos presentes en la región -Siluriano, Devoniano, Carbonífero, Margas irisadas (Keuper), Jurásico (Lías), de la Creta, Numulítico y Terciario, Diluvial y Aluvial- optando
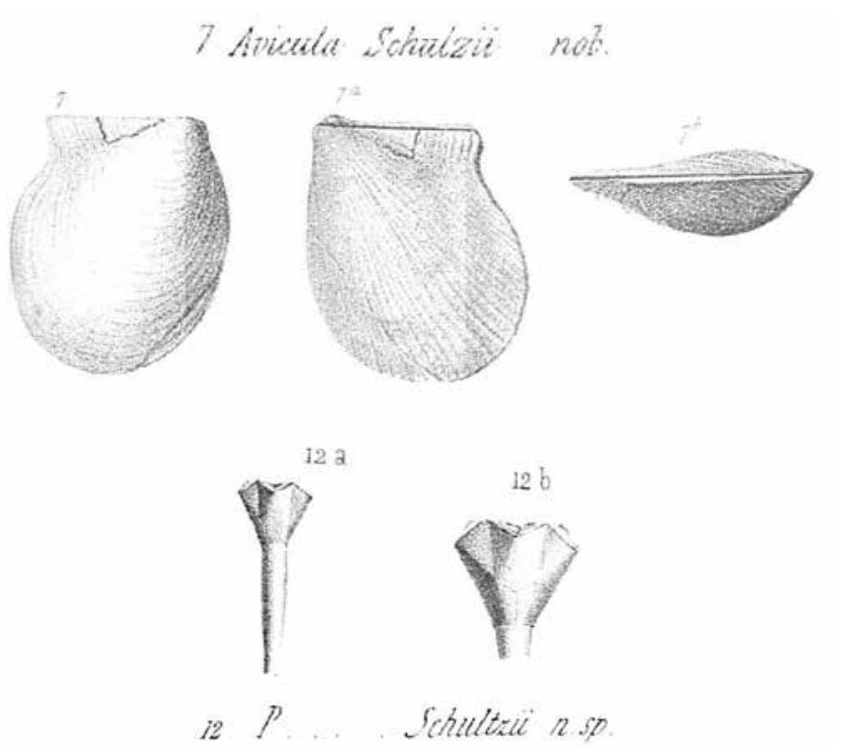

Figura 7. Fósiles dedicados a Schulz por Verneuil y d'Archiac y por Verneuil y Barrande (BSG, Tome XII, 1er. série, p. 964 y Tome II, 2e. série, p. 472).

Figure 7. Fossils dedicated to Schulz by Verneuil and d'Archiac and by Verneuil and Barrande. por incluir los distintos grupos de terreno plutónico que había reconocido en los apartados correspondientes a las formaciones en las que asomaban. En cada sección describió la clase, posición y aspecto de las rocas comprendidas, la fisonomía orográfica que presentaba el terreno en conjunto, y lo que, desde su punto de vista, podía ofrecer a la agricultura, a la industria y al comercio. Todos los rasgos generales de la estructura geológica asturiana, así como multitud de pequeños detalles significativos, quedaron consignados gráficamente en su Mapa Geológico de la Provincia de Oviedo, complementado con una lámina con cortes geológicos a distintas escalas, y dos vistas de los Picos de Europa. Con ello, Schulz culminaba su tarea científica, gracias a la cual el noroeste peninsular pudo disponer de una magnífica cartografía geológica casi al mismo tiempo que los países más avanzados del continente (Álvarez, 2004).

\section{Memorias en colaboración con Adrien Paillette}

Schulz y Paillette compartían una gran pasión por la geología, la minería y la metalurgia, por lo que al coincidir ambos en Asturias esta afinidad devino pronto en camaradería. Prueba de ello es que en varios de sus artículos se pone de manifiesto el fluido contacto que mantuvieron intercambiando datos $y$ consultas, o contrastando criterios, no siempre exentos de discrepancias. En 1849, su ya prolongada colaboración cristalizó en la redacción de una memoria conjunta titulada Notice sur une pyrite stannifère (Ballesterosite) et sur quelques gisements d'etain en Espagne, que Paillette presentó personalmente ante la Société Géologique de France en la sesión celebrada el 19 de noviembre ( $B S G$, Tomo VII, $2^{\text {a }}$ serie, pp. 16-24) (Fig. 8).

M. Paillette donne lecture du Mémoire suivant :

Notice sur une pyrite stannifère (ballestèrosite) et sur quelques gisements d'étain en Espagne, par MM. Guillaume Schulz, inspecteur général, et Adrien Paillette, ingénieur des mines.

Asturies, septembre 1849.

Introduction. - Parmi les pays qui jusqu’à présent ont fourni à la science le plus de minéraux et surtout de minerais d'étain, on ne voit figurer que fort rarement la péninsule Ibérique..., et pourtant elle est célèbre à plus d'un titre.

Figura 8. Presentación de la noticia sobre una pirita estañífera (BSG, Tome VII, 1er. série, p. 16).

Figure 8. Presentation of the news about a stanifer piryte. 
Con este trabajo, los dos ingenieros notificaban a la comunidad científica el hallazgo de lo que consideraban un nuevo mineral -pirita stannifera- en los esquistos arcillosos de las cercanías de Ribadeo y de Mondoñedo (Lugo), al que denominaron ballesterosita en honor del ministro de Hacienda que había impulsado la Ley de Minas de 1825. A modo de introducción incluían un comentario sobre los yacimientos de estaño existentes en Galicia y una completa descripción de las antiguas e inmensas labores mineras de Salabe y Ablaneda en Asturias. A partir de las referencias de los autores clásicos sobre los minerales explotados al norte de la Lusitania (Portugal), supusieron que estas últimas explotaciones habrían tenido por objeto, "con fuertes probabilidades", la obtención de dicho metal, idea que ya había sido sugerida por Schulz en sus primeras obras. Siendo el estudio de los vestigios de la minería antigua, otro punto de interés común entre ambos (Llaneza y González-Pumariega, 2007), anunciaban igualmente que en una próxima memoria que tenían preparada desde hacía ya mucho tiempo y de la que Paillette había dado noticia al duque de Glücksberg (1780-1860) en un informe sobre los yacimientos auríferos de España, tratarían de otras muchas minas de oro del Principado.

Seguidamente exponían las circunstancias del descubrimiento del mineral por parte de un herrero y concretaban la descripción mineralógica, cristalográfica y analítica de las muestras recibidas, indicando que el metal obtenido al caldear las pizarras que contenían la ballesterosita presentaba un alto contenido en estaño $(80 \%)$. Pese a todo, y dada la escasez de las muestras, señalaban que los ensayos realizados necesitaban ser comprobados y admitían la posibilidad de que el producto fuese "un accidente enteramente local».

Aun así, los esperanzadores resultados de los análisis practicados habían animado a varios particulares a realizar algunos registros mineros sobre mineral de estaño en la zona del descubrimiento. Esto llevó a que el 18 de octubre de 1849 -un mes antes de la presentación de la Noticia de Schulz y Paillette ante la Société Géologique de France- el ingeniero Agustín Martínez Alcíbar (1810-1872), que se encontraba al cargo de la subinspección de minas de Galicia creada a finales de 1845 , remitiera un informe a la inspección del distrito en el que exponía que el metal obtenido a partir de una especie mineralógica aún por determinar, era una aleación de plomo y estaño en la que, según sus cálculos, éste último intervendría en una proporción de un $25 \%$ del peso, por lo que proponía el nombre de Plumbostannita para dicha mena.

Paillette tuvo conocimiento de esta comunicación a su regreso de Francia, redactando de inmediato una nota complementaria a la Notice que fue leída el 17 de diciembre de aquel mismo año en la sociedad ( $B S G$, Tomo VII, $2^{\text {a }}$ serie, pp. 74-75). En ella se reafirmaba en su creencia de que la pirita era estannífera y manifestaba su esperanza de que nuevos ensayos permitieran demostrar que "la ballesterosita sería o se aproximaría a un estanno-sulfuro de plomo».

Pero a pesar de la opinión "tan respetable y de tanto peso" de su eminente colega y de su superior jerárquico, Martínez no se arredró, y preparó una réplica histórica-científica al contenido de la Notice que apareció publicada en junio de 1850 en la recién estrenada Revista Minera (Tomo I, pp. 33 a 50). Apoyado en sólidos argumentos, expuso que los antiguos laboreos estanníferos mencionados por Schulz y Paillette eran, en realidad, explotaciones sobre minerales auríferos de criaderos en roca firme, o criaderos hipogénicos. Además, sostuvo que la sustancia que realmente merecía el nombre de plumbostannita era muy diferente a la ballesterosita, y que debía ser el estanno-sulfuro de plomo cuya existencia había previsto el francés. A la vista de ello, concluía planteando elegantemente la posibilidad de que las muestras ensayadas por aquellos no hubieran estado completamente limpias.

Tanto Schulz como Paillette debieron asumir las objeciones a su memoria, puesto que ninguno de ellos redactó una contrarréplica, pero todo parece indicar que la controversia suscitada contribuyó de forma decisiva a que el germano no se corresponsabilizara finalmente con su colega francés de la monografía sobre la minería aurífera que habían anunciado y que con el título de Recherches sur l'histoire et les conditions de gisement des mines d'or dans le nord de l'Espagne fue presentada dos años después a la Société Géologique de France por Paillette (BSG, Tomo IX, $2^{\text {a }}$ serie, sesión del 21 de junio de 1852, pp. 482-504), apareciendo asimismo parcialmente traducida en 1853 en la Revista Minera (Tomo IV, pp. 450-457, 479483 y 510-514). No obstante, éste agradecería la participación de su querido compañero en el trazado de dos mapas en los que se representaba la posición de las antiguas labores romanas en Galicia y en Asturias, que constituyen las primeras representaciones cartográficas temáticas dedicadas a la minería antigua aparecidas en España (Fig. 9). Y también demostraría no guardar ningún resentimiento hacia su «amigo Alcíbar", al considerar sus procedimientos para ensayar minerales de oro como unos de los mejores.

\section{Otras memorias remitidas a la Société Géologique de France}

Tras el retorno en julio de 1854 de los progresistas al poder, el militar Francisco Luján (1798-1867) fue 


\section{CROQuIS GÉxÉr.}

de la position topogrophique des ancienurs Mtuen de losuest des Astaries.

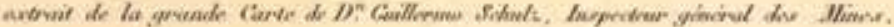

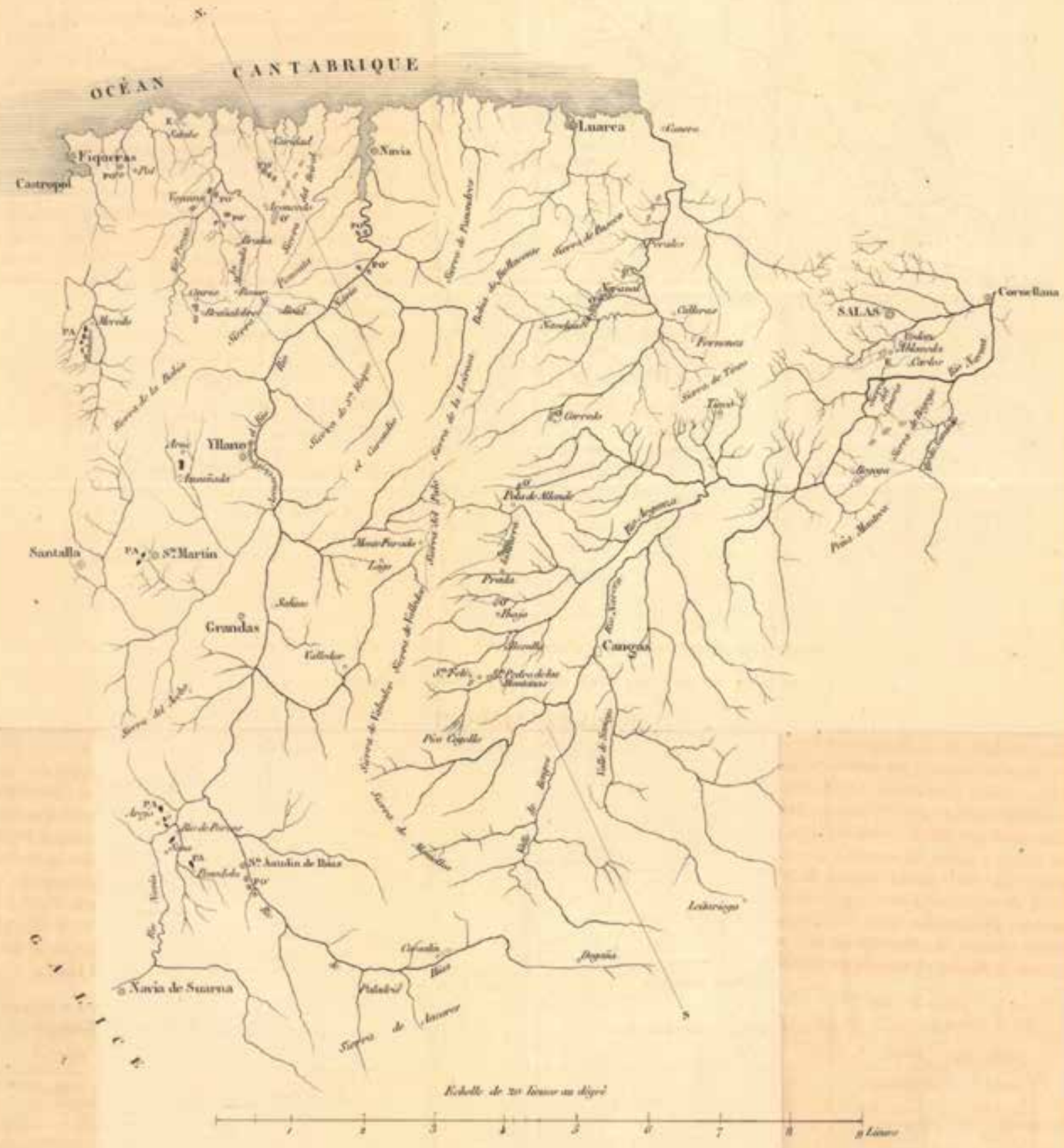

Figura 9. Mapa sobre la minería antigua de oro en Asturias compuesto por Schulz [Revista Minera, Tomo IV (1854), p. 514]. Figure 9. Map composed by Schulz to show the location of the old gold mines in Asturias. 
nombrado ministro de Fomento, por lo que al mes siguiente Schulz fue designado para reemplazarle en la presidencia de la Comisión para la Carta Geológica de Madrid y General del Reino, cargo que añadido a la dirección de la Escuela Especial de Ingenieros de Minas que ya venía ejerciendo desde mayo, determinó que recayeran sobre él la totalidad de las jefaturas unipersonales asignadas al Cuerpo de Minas.

Aprovechando esta coyuntura, así como la intención del nuevo gabinete de procurar modernizar la economía del país, Schulz promovió el estudio intensivo de las cuencas carboníferas peninsulares (RR.OO. del 27 de marzo de 1854). Dos años después y para dar a conocer los resultados de dichas investigaciones, redactó un artículo titulado Explotación de la hulla y del hierro en España y dibujó el Mapa Carbonífero de España y Portugal, a escala 1/2.500.000, en el que reseñó la situación de los principales grupos de hulla, lignito y turba conocidos. A modo de conclusión, lamentaba que siendo España un país rico en elementos de prosperidad, tuviera que importar carbón, hierro y toda clase de herramientas, por lo que hacía ver la necesidad de "confesar nuestra pereza y sacudirla" para imitar el ejemplo de otras naciones que con menos recursos habían conseguido desarrollarse y hacerse poderosas. Pero para ello resultaba fundamental el concurso de inversores extranjeros, por lo que para lograr una amplia difusión de su escrito lo remitió tanto a la Revista Minera (Tomo VII, pp. 10-17) como a la Société Géologique de France, anunciándose en esta última su recepción en la sesión del 5 de mayo de 1856 (BSG, Tomo XIII, $2^{\text {a }}$ serie, p. 586).

Por otra parte, a pesar de la penuria de medios con la que tuvo que afrontar la dirección del ambicioso conjunto de trabajos de carácter geográfico y naturalista encomendados a la Comisión del Mapa Geológico, con unos años de retraso consiguió publicar sendas memorias con el resumen de las actividades verificadas en 1854 y 1855 por sus secciones geológico-paleontológica, geológico-mineralógica, geográfico-meteorológica, botánica y zoológica (Rábano, 2015). Ambas fueron igualmente enviadas a la Société Géologique de France, recibiéndose, respectivamente, en las sesiones del 1 de diciembre de 1856 ( $B S G$, Tomo XIV, $2^{\text {a }}$ serie, p. 206) y del 7 de junio de 1858 ( $B S G$, Tomo XV, $2^{\text {a }}$ serie, p. 548 ).

\section{El papel de Schulz como "anfitrión" de la Société Géologique de France}

El artículo 4 del Reglamento constitutivo y administrativo de la Société Géologique de France establecía que para la incorporación de nuevos socios, estos debían ser presentados por dos miembros, firmar su adhesión al citado reglamento y ser proclamados por el presidente. Siendo Schulz uno de los primeros socios residente en España, participó también en la adhesión de algunos candidatos, figurando entre ellos varios de sus mejores amigos.

En este sentido, el primer aspirante a ingresar presentado por Schulz, junto con el entonces secretario d'Archiac, fue el profesor de química aplicada a las artes León Pérez de Salmeán (1810-1893), acontecimiento que tuvo lugar en la sesión del 2 de abril de 1838 (BSG, Tomo IX, $1^{\text {a }}$ serie, p. 227). Salmeán había iniciado sus estudios de ciencias en la cátedra de química docimástica de la Dirección General de Minas. De allí pasó al Museo de Ciencias Naturales y después al Conservatorio de Artes y al Jardín Botánico. También estudió en la Escuela de Farmacia de Madrid, llegando a ser el primer director de una fábrica de productos químicos establecida en la capital. Nombrado catedrático de la Universidad de Oviedo por real orden del 1 de diciembre de 1833, tomó posesión de su destino el 2 de noviembre de 1835. De la mano del germano, en 1842 participó como socio en una empresa minera que registró varias pertenencias de carbón y de hierro en varios concejos asturianos, y al año siguiente en la creación de la Sociedad Especial Unión Asturiana para la explotación de minas de cinabrio, galena argentífera y calamina. En 1866 accedió al cargo de rector de la Universidad de Oviedo, siendo reelegido de nuevo en 1868 y 1886 . Schulz recurrió a él para presentar a los demás interesados que recabaron su apoyo con el fin de incorporarse a la sociedad.

Así, el siguiente miembro apadrinado por ambos fue el ingeniero de minas valón Jean Louis Armand Nagelmackers (1808-1891), que fue admitido en la sesión del 21 de junio de 1841 (BSG, Tomo XII, $1^{\text {a }}$ serie, p. 369). Nagel -apelativo con el que también se le conocía- se había graduado en la École des Mines de Lieja en 1830 y tres años después fue contratado por su profesor Adolphe Lesoinne para dirigir las explotaciones de la recién creada Real Compañía Asturiana de Minas, siendo el responsable de introducir en la región los modernos métodos de laboreo. La estrecha relación que mantuvo con Schulz en aquellos años fomentó una relación de camaradería, por lo que en 1838 recabó su intermediación para pasar a ejercer la dirección técnica de la Compagnie de Minas d'Espagne constituida por Alejandro Aguado. Tras la muerte de éste, en 1843 se hizo cargo de las minas de Santo Firme (Llanera), propiedad del financiero madrileño José Safont (1803-1861), que posteriormente fueron transferidas a la Compañía Anglo-Asturiana, integrándose en sus cuadros técnicos. También participó 
en la creación de la Unión Asturiana, la Sociedad Minera Gijonesa-embrión de la Fábrica de Vidrios de Gijón- y en la Fábrica de Vidrios de Avilés. En 1851 regresó a Bélgica, siendo nombrado vicecónsul español en Lieja. En su país natal participó en la Société Anonyme des Hautes Fourneaux et Fonderies de Dolían y en la Société des Mines et Fonderies de Zinc de la Vieille-Montagne. En 1857, fue nombrado cabaIlero de la Real Orden de Carlos III (González, 2014).

Casi cinco años después -sesión del 16 de febrero de 1846- Schulz y Salmeán presentaron a los jóvenes ingenieros del Cuerpo de Minas Policarpo Cía (1817-1867) y Joaquín Luaces (¿?-1847) (BSG, Tomo III, $2^{\text {a }}$ serie, p. 267). Cía fue el alumno más brillante de la primera promoción de la Escuela de Ingenieros de Minas de Madrid, egresada en 1838. Su primer destino fueron las minas de Almadén, donde también impartió clases en la reorganizada Escuela para Capataces. En 1844, y después de una breve estancia en las minas de Linares (Jaén), pasó a desempeñar la secretaría de la inspección de Asturias y Galicia, donde coincidió unos meses con Schulz. Al año siguiente fue comisionado para realizar una visita de inspección a las minas de Huesca y Navarra. Tras una baja por razones administrativas, en 1846 se reincorporó al servicio, siendo destinado, a petición propia, a la provincia de Puerto Príncipe (Cuba), si bien antes realizó una visita científica a los establecimientos metalúrgicos de Swansea (Inglaterra). En 1850 regresó a la Península, integrándose en el claustro de profesores de la Escuela de Minas de Madrid y haciéndose cargo de varias cátedras. Dos años después fue comisionado para estudiar la tecnología minera de Suecia, Noruega y Finlandia. A partir de 1854 compatibilizó su función docente con la dirección de una de las minas de plata de Hiendelaencina (Guadalajara). En 1862 fue nombrado director de la Escuela, aunque su estado de salud le obligó a solicitar la jubilación voluntaria al cabo de dos años.

Luaces ingresó en 1841 en la escuela especial del ramo. A la conclusión de sus estudios superiores, en 1844 fue nombrado aspirante segundo del Cuerpo de Ingenieros de Minas, siendo destinado a la Inspección de Riotinto. Un año después publicó una memoria en el Boletín Oficial de Minas titulada Descripción del procedimiento que se sigue en Los Planes, dependencia del establecimiento de Riotinto, para la extracción del cobre que contienen los vitriolos y tierras de sus minas y el aprovechamiento de la caparrosa. En 1846 pasó a servir como ayudante segundo en la inspección de la provincia de León, donde falleció prematuramente al año siguiente.

Por último, en la sesión del 6 de enero de 1851 los avalados fueron Francisco Antonio de Elorza
(1798-1873) y Adolphe Jean Charles Desoignie (18161898) (BSG, Tomo VIII, $2^{\text {a }}$ serie, p. 144). Elorza ingresó en el Real Colegio de Artillería en 1811. Durante el Trienio Constitucional ejerció las funciones de jefe de Estado Mayor, viéndose obligado a exiliarse con el restablecimiento del régimen absolutista. No obstante, aprovechó esta circunstancia para estudiar las nuevas técnicas de fundición del hierro en diversos centros de enseñanza y establecimientos industriales de Francia, Bélgica, Alemania, Piamonte e Inglaterra. En 1829 obtuvo permiso para regresar a España a dirigir, como ingeniero civil, tres ferrerías en Marbella, Málaga y EI Pedroso (Sevilla), tarea con la que contribuyó decisivamente al arranque de la moderna siderurgia española. Incorporado en 1843 al servicio militar activo, se le encomendó restablecer la Fábrica de Armas de Trubia (Asturias) y más tarde modernizar la de Oviedo, introduciendo en ambas numerosos adelantos técnicos. La amistad que trabó con Schulz en Andalucía y consolidó después en Asturias, terminó fraguando en un pequeño proyecto industrial, al participar en 1852 con el germano en la creación de una fábrica de tejidos en Vivero (Lugo). En 1863 Elorza fue nombrado brigadier y destinado a la Junta Superior Facultativa de Artillería. Al año siguiente ascendió a mariscal de campo.

Tras formarse como ingeniero de minas en la Université de Liège, Desoignie llegó a Asturias en 1838 para sustituir a Nagel en la dirección técnica de la Real Compañía Asturiana de Minas, cargo que desempeñó hasta 1853, estableciendo a lo largo de estos años una gran amistad con Schulz. Arraigado en la región, el técnico belga permaneció en ella hasta su muerte, creando una empresa de minas propia y realizando algunos trabajos de ingeniería para el Ayuntamiento de Avilés, como la red de abastecimiento de aguas o el diseño del parque de la Alameda Vieja. En 1863, y siguiendo unos planteamientos similares a los expuestos por Schulz dos décadas antes, propuso un proyecto de red ferroviaria para la región que enlazara la cuenca carbonífera central con los puertos de Gijón, Avilés y Luanco. Considerándolo como uno de sus amigos más afectuosos y más enterado de sus asuntos y negocios, el germano le designó en 1877 como albacea testamentario suplente.

\section{Conclusiones}

La relación de Schulz con la Société Géologique de France solo ha sido citada someramente por algunos de sus biógrafos para referir su condición de miembro de la misma y mencionar la publicación de sus artículos en el Bulletin. Sin embargo, el vínculo que el 
ingeniero de minas y funcionario español estableció con la asociación gala sería de gran importancia para el desarrollo de su labor científica y profesional. Por un lado, al incorporarse tempranamente como socio a la misma, se aseguró el acceso indispensable a los rápidos avances experimentados por la geología en un momento en el que la disciplina se estaba consolidando como ciencia independiente. Gracias a ello, pudo depurar y adaptar sus conocimientos y observaciones a los principios más modernos establecidos en el seno de la comunidad científica, abordando así con éxito la elaboración de las descripciones geológicas y los mapas petrográficos de las provincias de Galicia y Asturias que se le habían encomendado. En justa correspondencia, Schulz remitió en primicia a la asociación los resultados de sus investigaciones, por lo que al dar a conocer la conformación geológica del noroeste peninsular contribuyó significativamente al progreso de dicha ciencia.

Pero además, como alto funcionario de la Junta Superior Facultativa de Minería, el germano también se encargó de promover el desarrollo del ramo, dando a conocer la abundancia de recursos minerales susceptibles de explotación en las regiones estudiadas, así como de ricos yacimientos de carbón y hierro en el conjunto de España. En este sentido, aprovechó la capacidad de difusión del Bulletin para acceder mediante sus artículos divulgativos a los inversores extranjeros, lo que sin duda estimuló la creación de muchas de las compañías mineras y siderúrgicas, participadas en gran medida por socios franceses $y$ belgas, que se instalaron en España a mediados del siglo XIX.

Schulz también contribuyó a los fines de la Société Géologique de France colaborando y compartiendo información con los miembros que visitaron nuestro país y promoviendo el ingreso de nuevos socios.

\section{Referencias}

Álvarez, E. 2000. Raíces mineras y metalúrgicas de la geología. Revista de Minas de la Universidad de Oviedo, 19-20, 21-26.

Álvarez, E. 2004. Filosofía de las ciencias de la tierra. El cierre categorial de la geología. Biblioteca Filosofía en español. Fundación Gustavo Bueno. Pentalfa ediciones, Oviedo, $355 \mathrm{pp}$.

Aragonés, E. 1999. Noticia de D. Ángel Vallejo y Villalón (1778-1840), comisionado para realizar estudios geológicos en Cataluña y encargado de formar el primer mapa geológico de España. Boletín Geológico y Minero, $110(5), 93-105$.

Boué, A. 1832. Résumé des progrès de la géologie en 1830 et 1831. Bulletin de la Société Géologique de France, II, 133-218.
Boué, A. 1861. Documents sur l'origine de la Société géologique de France (extrait). Bulletin de la Société Géologique de France, XVIII, 130-132.

Cuvier, G. y Brongniart, A. 1811. Essai sur la géographie minéralogique des environs de Paris, avec une carte géognostique, et des coupes de terrain. Baudouin, imprimeur de l'Institut de France. 278 pp.

Ellenberger, F. 1982. Les premieres cartes geologiques en France: projets et realisations. Travaux du Comité Français d'Histoire de la Géologie, Première série, 29/01/2016, http://www.annales.org/archives/cofrhigeo/premieres-cartes-geologiques.html

Ezquerra, J. 1839. Elementos de laboreo de minas, precedidos de algunas nociones sobre geognosia y la descripción de varios criaderos de minerales, tanto de España como de otros reinos de Europa. Imprenta de Don Salvador Albert, Madrid. 448 pp.

Ezquerra, J. 1844. Datos y observaciones sobre la industria minera, con una descripción característica de los minerales útiles, cuyo beneficio puede ser objeto de las empresas. Imprenta de D. Antonio Yenes, Madrid, 353 pp.

Ezquerra, J., García, F. de S., Amar, R. y Bauzá, F. 1831. Minas de carbón de piedra de Asturias. Reconocimiento hecho de orden del Rey N. Sr. por una Comisión de Facultativos. Imprenta de D. José del Collado, Madrid, $62 \mathrm{pp}$.

Fernández de Castro, M. 1874. Notas para un estudio bibliográfico sobre los orígenes y estado actual del mapa geológico de España. Boletín de la Comisión del Mapa geológico de España, l, 17-168.

García-Cortés, Á. 2005. La cartografía geológica en España desde Guillermo Schulz hasta la actualidad. En: Rábano, I. y Truyols, J. (Eds.), Miscelánea Guillermo Schulz (18051877). Cuadernos del Museo Geominero, 5. Instituto Geológico y Minero de España, Madrid, 153-177.

González, L.A. 2014. Armand Nagelmackers, el pionero olvidado de la industrialización asturiana. La Nueva España 15/06/2014.

González, L.A. 2015. Las empresas de Alejandro Aguado, marqués de las Marismas, en Asturias: camino carbonero y la compañía minera. Los inicios del desarrollo minero y siderúrgico de Asturias (1775-1860). Real Instituto de Estudios Asturianos, Oviedo, 96-127.

González-Pumariega, P. 2011. Guillermo Schulz y su obra. Aportaciones a la modernización de Asturias. Tesis doctoral, Universidad de Oviedo, 693 pp.

Hausmann, J.F.L. 1831. De Hispaniæ Constitutione geognostica. Typis Dieterichianis, Gotinga, 24 pp.

Lacroix, A. 1928. Notice historique sur le troisième fauteuil de la section de minéralogie. Séance publique annuelle du lundi 17 décembre 1928. Institut de France, Académie des Sciences. Gauthier-Villars et Cie, 86 pp.

Laurent, G. 2006. Paléontologie et évolution: la Société géologique de France, "espace de liberté" (1830-1860). Travaux du Comité français d'Histoire de la Géologie, 20, 1-11.

Llaneza, L.J. 2010. Aproximación a Paillette, personalidad relevante en el inicio del desarrollo minero e industrial asturiano. Libro de actas. VIII Congreso Internacional 
sobre Patrimonio Geológico y Minero, Oviedo. SEDPGYM, 293-300.

Llaneza, L.J. y González-Pumariega, P. 2010. Paillette y Schulz, adelantados de la arqueología minera asturiana. Libro de actas. VIII Congreso Internacional sobre Patrimonio Geológico y Minero, Oviedo. SEDPGYM, 301-309.

Lobé, G. 1842. Mi segundo viaje a Europa (y especialmente a España) en los años de 1840 a 1842. Tomo IV. Imprenta de Alegría y Charlain, Madrid, 330 pp.

Madoz, P. 1845. Diccionario Geográfico-EstadísticoHistórico de España y sus posesiones de Ultramar. Vol. I. Establecimiento tipográfico de P. Madoz y L. Sagasti, Madrid, 86.

Maestre, A. 1845. Noticia geológica de la Sierra de Gádor e informe sobre el establecimiento de un socavón general en la misma. Boletín Oficial de Minas, IV (30), 398.

Maffei, E. 1877. Necrología. D. Guillermo Schulz. Revista Minera, Científica, Industrial y Mercantil, XXVIII, 178-180.

Ordaz, J. 1978. La geología en España en la época de Guillermo Schulz (1800-1877). Trabajos de Geología, 10, 21-35.

Puche, O. y Ayala-Carcedo, F.J. 2001. Guillermo P.D. Schulz y Schweizer (1800-1877): su vida y su obra en el bicentenario de su nacimiento. Boletín Geológico y Minero, 112 (1), 105-122.

Rábano, I. 2015. Los cimientos de la Geología. La Comisión del Mapa Geológico de España (1849-1910). Instituto Geológico y Minero de España, Madrid, 329 $\mathrm{pp}$.

Truyols, J. y Schroeder, R. 2005. Nuevas investigaciones sobre la fecha de nacimiento y la juventud de Guillermo Schulz. En: Rábano, I. y Truyols, J. (Eds.), Miscelánea Guillermo Schulz (1805-1877). Cuadernos del Museo Geominero, 5. Instituto Geológico y Minero de España, Madrid, 13-28.

Vidal, J.R. 2005. La obra de Guillermo Schulz en Galicia. En: Rábano, I. y Truyols, J. (Eds.), Miscelánea Guillermo Schulz (1805-1877). Cuadernos del Museo Geominero, 5. Instituto Geológico y Minero de España, Madrid, 79-108.

Viquesnel, A. 1844. Statistique administrative de la Société Géologique de France depuis l'epoque de sa fondation, en 1830, jusqu'au 31 décembre 1843. Imprimerie de Bougogne et Martinet, París, 54 pp.

Vitar, M.B. 2010. Lorenzo Gómez Pardo y Ensenyat. Correspondencia de Guillermo Schulz (1835-1843). Iberoamericana-Vervuert, Madrid, 223 pp.

Recibido: julio 2016

Revisado: agosto 2016

Aceptado: septiembre 2016

Publicado: marzo 2017 
